\title{
Ordering multiple soft gluon emissions using SCET with Glauber operators
}

\author{
Jeffrey Forshaw, Jack Holguin, and Aditya Pathak® \\ University of Manchester, School of Physics and Astronomy, Manchester M13 9PL, United Kingdom
}

(Received 8 September 2021; accepted 12 November 2021; published 29 November 2021)

\begin{abstract}
In QCD, the amplitude for multiple soft gluon emissions has been shown to exhibit a form of coherence, wherein one-loop corrections are rendered IR finite by the transverse momentum of the real emissions. Using a sequence of soft-collinear effective theories (SCET) with Glauber operators, we present a compact and elegant derivation of this property. Our derivation highlights an important role played by the Lipatov vertex. The EFT approach provides increased physical intuition and, for the first time, a clear road map for how this result can be extended to higher loop orders.
\end{abstract}

DOI: 10.1103/PhysRevD.104.L091501

\section{INTRODUCTION}

There is a remarkable property in perturbative $\mathrm{QCD}$, so far proven only at one-loop accuracy, whereby the amplitude for soft gluon emission off a generic hard process can be organized in such a way that the infrared (IR) divergences associated with the loop integrals can be tamed [1]. Specifically, the amplitude for multigluon emission can be written as a sum over Markovian chains of real emissions with the loop inserted into the chain at all possible places. The associated loop integrals are all rendered IR finite by the transverse momentum of the next emission in the chain. As a result, only the case where the loop integral occurs at the end of the chain is IR divergent.

The original derivation of this result $[1,2]$ involved a complicated sum over many Feynman diagrams, which makes the extension to higher orders a formidable task. In this paper, we rederive this result in the framework of soft collinear effective theory (SCET) [3-7] with Glauber potential operators [8]. The use of SCET drastically simplifies the analysis with far fewer diagrams and a clear physical picture. In other words, the calculation in SCET is organized in such a way that the appropriate grouping of the QCD diagrams is automatic. This should pave the way to an all-loops calculation in the future. Our use of SCET is also novel in the sense that we have used it as a tool for a fixedorder calculation in a multiscale problem. We keep the discussion brief and refer the reader to a companion paper [9] for explicit details of the calculation and other interesting related results.

Published by the American Physical Society under the terms of the Creative Commons Attribution 4.0 International license. Further distribution of this work must maintain attribution to the author(s) and the published article's title, journal citation, and DOI. Funded by SCOAP ${ }^{3}$.
Soft gluon factorization is an important property of QCD (e.g., see [10-15]). Accordingly, the amplitude for the emission of $N$ soft gluons off an $n$-parton hard scattering process, $\left.\left.\mid M_{N}\right]=\mid \mathcal{M}\left(q_{1}, \ldots, q_{N}, p_{1}, \ldots p_{n}\right)\right]$, can be written as a product of soft gluon emission operators acting on a dressed hard scattering vector:

$$
\left.\left.\mid M_{N}\right] \simeq\left(g \mu^{\epsilon}\right)^{N} \mathbf{J}\left(q_{N}\right) \cdots \mathbf{J}\left(q_{1}\right) \mid M_{0}\right],
$$

where $q_{i}$ is a soft gluon momentum and $p_{i}$ is a hard parton. The soft momenta have a hierarchy so that $q_{i}$ is more soft than $q_{i-1}$. The bracket notation indicates vectors in $\mathrm{SU}(3)$ color space. The hard scattering vector and the soft gluon operators have loop expansions [12]:

$$
\begin{aligned}
\left.\mid M_{0}\right] & \left.\left.=\mid M_{0}^{(0)}\right]+\mid M_{0}^{(1)}\right]+\cdots \\
\text { and } \quad \mathbf{J}(q) & =\mathbf{J}^{(0)}(q)+\mathbf{J}^{(1)}(q)+\cdots,
\end{aligned}
$$

where the superscripts denote the loop order. At tree level,

$$
\begin{aligned}
\mathbf{J}^{(0)}\left(q_{m+1}\right) & =\sum_{j=1}^{n+m} \mathbf{T}_{j} \frac{p_{j} \cdot \varepsilon}{p_{j} \cdot q_{m+1}}=\sum_{j=1}^{n+m} \mathbf{d}_{i j}^{(0)}\left(q_{m+1}\right), \\
\mathbf{d}_{i j}^{(0)}(q) & =\mathbf{T}_{j}\left(\frac{p_{j} \cdot \varepsilon}{p_{j} \cdot q}-\frac{p_{i} \cdot \varepsilon}{p_{i} \cdot q}\right),
\end{aligned}
$$

where $\varepsilon$ is the polarization vector of the emitted gluon. For later convenience, we have expressed the tree level soft gluon current $\mathbf{J}^{(0)}$ in terms of a sum over dipole emission operators $\mathbf{d}_{i j}^{(0)}$. Because of color conservation this relation holds for any choice of parton $i$ (the free index not summed over). Note also that the amplitude for emission of the $q_{m+1}$ gluon involves all the hard momenta $\left\{p_{1}, \ldots p_{n}\right\}$ as well as the soft momenta $\left\{q_{1}, \ldots, q_{m}\right\}$. Thus we have defined $p_{n+m} \equiv q_{m}$ in Eq. (3). At one loop, 


$$
\begin{aligned}
\mathbf{J}^{(1)}\left(q_{m+1}\right) & =\sum_{j=1}^{n+m} \sum_{k=1}^{n+m} \mathbf{d}_{j k}^{(1)}\left(q_{m+1}\right), \\
\mathbf{d}_{i j}^{(1)}(q) & =\frac{\alpha_{s}}{2 \pi} \frac{c_{\Gamma}}{\epsilon^{2}} \mathbf{T}_{q} \cdot \mathbf{T}_{i}\left(\frac{e^{-\mathrm{i} \pi \tilde{\delta}_{i j}}}{e^{-\mathrm{i} \pi \tilde{\delta}_{i q}} e^{-\mathrm{i} \pi \tilde{\delta}_{j q}}} \frac{4 \pi \mu^{2}}{\left(q_{\perp}^{(i j)}\right)^{2}}\right)^{\epsilon} \mathbf{d}_{i j}^{(0)}(q),
\end{aligned}
$$

with $c_{\Gamma}=\frac{\Gamma^{3}(1-\epsilon) \Gamma^{2}(1+\epsilon)}{\Gamma(1-2 \epsilon)}$. In contrast with Eq. (3), the expression for $\mathbf{J}^{(1)}$ involves a sum over both indices of $\mathbf{d}_{j k}^{(1)}$, and color conservation cannot be used to simplify it any further. The corresponding one-loop hard scattering vector is

$$
\left.\left.\mid M_{0}^{(1)}\right]=\sum_{i=2}^{n} \sum_{j=1}^{i-1} \mathbf{I}_{i j}\left(0, \omega_{i j}\right) \mid M_{0}^{(0)}\right]
$$

where $\omega_{i j}=2 p_{i} \cdot p_{j}$ and the loop correction is given by

$$
\mathbf{I}_{i j}\left(0, \omega_{i j}\right)=\frac{\alpha_{s}}{2 \pi} \frac{c_{\Gamma}}{\epsilon^{2}} \mathbf{T}_{i} \cdot \mathbf{T}_{j}\left(e^{\mathrm{i} \pi \tilde{\delta}_{i j}} \frac{4 \pi \mu^{2}}{\omega_{i j}}\right)^{\epsilon}
$$

where $\tilde{\delta}_{i j}=1$ if partons $i$ and $j$ are either both incoming or both outgoing and $\tilde{\delta}_{i j}=0$ otherwise. In this way of approaching things, both the soft gluon emission operators and the hard scattering vector are infrared (IR) divergent (i.e., they have $1 / \epsilon$ poles).

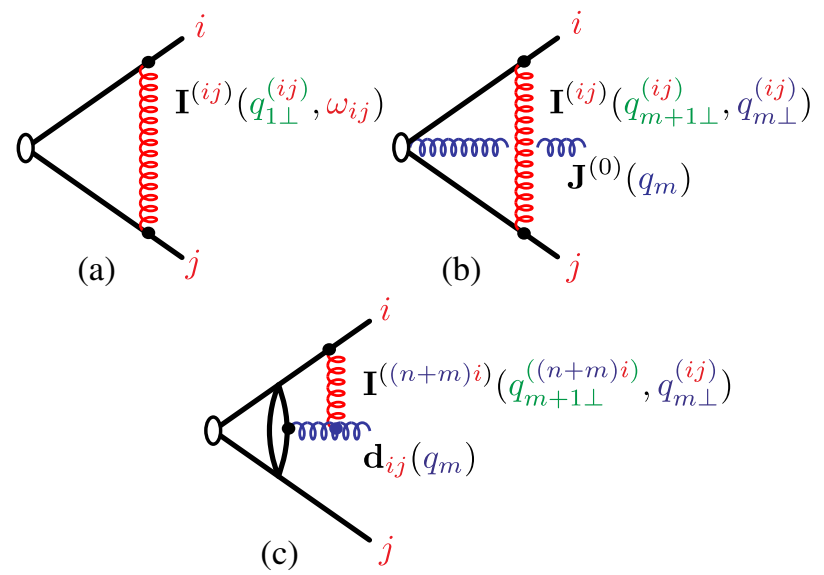

FIG. 1. Relation of limits of the virtual loop insertions to the adjacent dipole emission momenta in the ordered soft gluon emission result. The graphs (a), (b) and (c) correspond to the three lines in Eq. (7).

Remarkably, at one-loop at least, this result for the amplitude can be rewritten as a chain of real emissions with a loop inserted at any point in the chain. Crucially, all of the loop integrals except for the last one are rendered finite since they are cutoff by the transverse momentum of the next emission in the chain (evaluated in an appropriate frame). Specifically, the one-loop amplitude with $N$ emissions can be written:

$$
\begin{aligned}
\left.\mid M_{N}^{(1)}\right]= & \left.\left(g \mu^{\epsilon}\right)^{N}\left(\prod_{k=1}^{N} \mathbf{J}^{(0)}\left(q_{k}\right)\right)\left(\sum_{i=2}^{n} \sum_{j<i} \mathbf{I}^{(i j)}\left(q_{1 \perp}^{(i j)}, \sqrt{\omega_{i j}}\right)\right) \mid M_{0}^{(0)}\right] \\
& \left.+\left(g \mu^{\epsilon}\right)^{N} \sum_{m=1}^{N}\left(\prod_{k=m+1}^{N} \mathbf{J}^{(0)}\left(q_{k}\right)\right)\left(\sum_{i=2}^{n+m-1} \sum_{j<i} \mathbf{I}^{(i j)}\left(q_{m+1 \perp}^{(i j)}, q_{m \perp}^{(i j)}\right)\right)\left(\prod_{\ell=1}^{m} \mathbf{J}^{(0)}\left(q_{\ell}\right)\right) \mid M_{0}^{(0)}\right] \\
& \left.+\left(g \mu^{\epsilon}\right)^{N} \sum_{m=1}^{N}\left(\prod_{k=m+1}^{N} \mathbf{J}^{(0)}\left(q_{k}\right)\right)\left(\sum_{i, j=1}^{n+m-1} \mathbf{I}^{((n+m) i)}\left(q_{m+1 \perp}^{((n+m) i)}, q_{m \perp}^{(i j)}\right) \mathbf{d}_{i j}^{(0)}\left(q_{m}\right)\right)\left(\prod_{\ell=1}^{m-1} \mathbf{J}^{(0)}\left(q_{\ell}\right)\right) \mid M_{0}^{(0)}\right]
\end{aligned}
$$

where

$$
\mathbf{I}_{i j}(a, b)=\frac{\alpha_{s}}{2 \pi} \mathbf{T}_{i} \cdot \mathbf{T}_{j} \frac{c_{\Gamma}}{\epsilon^{2}}\left[\left(\frac{4 \pi \mu^{2}}{b^{2}}\right)^{\epsilon}\left(1+\mathrm{i} \pi \epsilon \tilde{\delta}_{i j}-\epsilon \ln \frac{\omega_{i j}}{b^{2}}\right)-\left(\frac{4 \pi \mu^{2}}{a^{2}}\right)^{\epsilon}\left(1+\mathrm{i} \pi \epsilon \tilde{\delta}_{i j}-\epsilon \ln \frac{\omega_{i j}}{a^{2}}\right)\right]+\operatorname{Re} \mathcal{O}\left(\epsilon^{0}\right)
$$

The $\perp$ subscript is defined so that $q_{\perp}^{(i j)}$ is the transverse momentum defined in the $i j$ zero momentum frame. Note that the real part of Eq. (7) can be obtained from the imaginary part by analytic continuation:

$$
\mathrm{i} \pi \epsilon \tilde{\delta}_{i j}-\epsilon \ln \frac{\omega_{i j}}{\mu^{2}}=\epsilon \ln \frac{\mu^{2}}{-\omega_{i j}} .
$$

The result can be understood by relating each of the three lines in Eq. (7) with the three diagrams shown in Fig. 1 [the color coding in Eq. (7) agrees with the colors in Fig. 1 and later in Eq. (21)]. The loop insertions are given by $\mathbf{I}_{i j}(a, b)$ in Eq. (8). The first argument represents the lower limit of the loop integral and it is determined by the next soft gluon emission in the chain (this gluon is not shown in the figure). The second argument represents the upper limit and it is determined by the previous soft gluon emission. In the first line, the upper limit is simply the hard scale (there are no prior emission). So long as $a, b>0$, one can safely take the 
limit $\epsilon \rightarrow 0$ in Eq. (8). The dipole frame in which loop cutoff momenta are evaluated carries special significance. In the first two lines it is just the frame defined by the two partons between which the virtual gluon is exchanged. The third line is interesting for it reveals that the gluon's transverse momentum is to be evaluated in the rest frame of its parent-dipole. Because of this, the individual dipole contributions cannot be summed over in this term, preventing the result from being written in terms of $\mathbf{J}$ operators.

\section{DERIVATION FROM GLAUBER-SCET}

It is sufficient for us to consider the imaginary part of the one-loop result in Eq. (7), which means we can focus solely on diagrams with a single Glauber exchange loop [8]. In overview, we use SCET to compute $\left.\operatorname{Im} \mid M_{2}^{(1)}\right]$ from which the generalization to $\left.\mid M_{N}^{(1)}\right]$ is reasonably simple. We exploit the hierarchy between the two soft emissions by defining two EFTs, $\mathrm{EFT}_{n}$ and $\mathrm{EFT}_{n+1}$. In both the EFTs the hard momenta $\left(p_{1}, \ldots p_{n}\right)$ define $n$ collinear directions. In $\mathrm{EFT}_{n+1}$ we treat the soft gluon $q_{1}$ as resolved by further restricting the fluctuations in its virtuality so that it may be considered as a new collinear mode. The virtuality of $q_{2}$ then sets the scale of soft fluctuations in $\mathrm{EFT}_{n+1}$. The hard scattering operator in $\mathrm{EFT}_{n+1}$, including $q_{1}$ as a collinear mode, is easily fixed based on symmetry considerations.

The operators necessarily come with Wilson coefficients which encode the UV physics, which we compute at oneloop. The Wilson coefficients carry dependence on the large momenta, $\omega_{i}=\bar{n}_{i} \cdot p_{i}=p_{i}^{-}$, in direction $n_{i}$ (with $\bar{n}_{i}$ being an auxiliary light like vector satisfying $\bar{n}_{i} \cdot n_{i}=2$ ). Crucially, we will see that the large momentum corresponding to the $q_{1}$ gluon, used in $\mathrm{EFT}_{n+1}$, is transverse momentum $q_{1 \perp}^{(i j)}$.

The hard scatterings in $\mathrm{EFT}_{n}$ and $\mathrm{EFT}_{n+1}$ are described by the operators,

$$
\begin{aligned}
& O_{n}^{\text {hard scatter }} \\
& =\int\left(\prod_{i=1}^{n} d \omega_{i}\right)\left[O_{n}^{(0)}\left(\left\{\omega_{i}, n_{i}\right\}\right)\left|\prod_{i=1}^{n} \mathbf{S}_{n_{i}}\right| \mathcal{C}_{n}\left(\left\{\omega_{i}\right\}, \mu\right)\right], \\
& O_{n+1}^{\text {hard scatter }} \\
& =\int\left(\prod_{i=1}^{n+1} d \omega_{i}\right)\left[O_{n+1}\left(\left\{\omega_{1}, n_{1}, \omega_{i}, n_{i}\right\}\right) \mid \mathcal{C}_{n+1}\left(\left\{\omega_{1}, \omega_{i}\right\}, \mu\right)\right] .
\end{aligned}
$$

where

$$
\begin{aligned}
& {\left[O_{n+1}\left(\left\{\omega_{1}, n_{1}, \omega_{i}, n_{i}\right\}\right) \mid\right.} \\
& =\left[O_{n}^{(0)}\left(\left\{\omega_{i}, n_{i}\right\}\right) \mid\left[g \sum_{i=1}^{n} \frac{n_{i} \cdot \mathcal{B}_{n_{1} \perp, \omega_{1}}^{(0) a}}{n_{i} \cdot q_{1}} \mathbf{T}_{i}^{a}\right]\left(\prod_{i=1}^{n} \mathbf{S}_{n_{i}}\right) \mathbf{S}_{q_{1}},\right.
\end{aligned}
$$

$\mathcal{B}_{n_{1} \perp, \omega_{1}}^{(0) a}$ is the gauge invariant building block [6] for the harder of the two soft gluons (i.e., corresponding to $q_{1}$ ). The notation indicates that it is perpendicular to $n_{1}$. Here the operators and the Wilson coefficients are dual-vectors and vectors in the color space. Off-shell modes have been integrated out via a BPS field redefinition [5] of the collinear fields to obtain soft Wilson lines in directions $n_{i}$, and the adjoint Wilson line $\mathbf{S}_{q_{1}}$ in the direction $n_{1}$. Consequently, the operators $O_{n, n+1}^{(0)}$ consist solely of collinear fields. $\left.\mid \mathcal{C}_{n}\left(\left\{\omega_{1}\right\}, \mu\right)\right]$ and $\left.\mid \mathcal{C}_{n+1}\left(\left\{\omega_{1}, \omega_{i}\right\}, \mu\right)\right]$ are the Wilson coefficients, they have a loop expansion for which we use the same notation as in Eq. (2).

For the general case of $n$ hard partons and $N$ soft emissions, the correspondence between QCD and $\mathrm{EFT}_{n}$ amplitudes is

$$
\begin{aligned}
& \left.\sum_{N}\left(g \mu^{\epsilon}\right)^{N} \mathbf{J}\left(q_{N}\right) \ldots \mathbf{J}\left(q_{1}\right) \mid \mathcal{M}\left(p_{1}, \ldots, p_{n}\right)\right] \\
& =\int\left(\prod_{i=1}^{n} d \omega_{i}\right)\left\langle\left\{p_{i}\right\},\left\{q_{j}\right\}\right| \\
& \left.\quad \times \mathrm{T}\left\{O_{n}^{(0)}\left(\left\{\omega_{i}, n_{i}\right\}\right) \prod_{i=1}^{n} \mathbf{S}_{n_{i}} e^{\mathrm{i} \int d^{4} x^{\prime} O_{G}\left(x^{\prime}\right)}\right\}|0\rangle \mid \mathcal{C}_{n}\left(\left\{\omega_{i}\right\}\right)\right],
\end{aligned}
$$

where $\left\{a_{i}\right\}$ is the set of color indices for the hard particles and $\left\{C_{j}\right\}$ the color indices for soft particles. Here, $O_{G}(x)$ consists of the Glauber operators from the SCET Glauber Lagrangian [8]. Note that

$$
\sum_{\left\{a_{i}\right\}}\left[\left\{a_{i}\right\} \mid O_{n}^{(0)}\left(\left\{\omega_{i}, n_{i}\right\}\right)=\left[O_{n}^{(0)}\left(\left\{\omega_{i}, n_{i}\right\}\right) \mid .\right.\right.
$$

By expanding the right-hand side of Eq. (12) to oneloop, we arrive at the result in Ref. [1] using the following four steps, corresponding in turn to the four sets of diagrams in Fig. 2.

(1) Expand Eq. (12) perturbatively at tree level and one loop for $N=0$ using $\mathrm{EFT}_{n}$ [Fig. 2(a)]. This fixes $\left.\mid \mathcal{C}_{n}^{(0)}\right]$ and the imaginary part of $\left.\mid \mathcal{C}_{n}^{(1)}\right]$.

(2) Compute $\left.\operatorname{Im} \mid M_{1}^{(1)}\right]$ by evaluating the matrix element for single-soft emission in $\mathrm{EFT}_{n}$ [Fig. 2(b)] and combining it with $\left.\mid \mathcal{C}_{n}\right]$ from step 1.

(3) Use the result from step 2 to calculate $\left.\mid \mathcal{C}_{n+1}^{(0)}\right]$ and $\left.\operatorname{Im} \mid \mathcal{C}_{n+1}^{(1)}\right]$ by computing the one-loop, no real emission graphs in $\mathrm{EFT}_{n+1}$ [Fig. 2(c)].

(iv) Calculate the one-loop single soft emission amplitude in $\mathrm{EFT}_{n+1}$ [Fig. 2(d)] and combine it with $\left.\operatorname{Im} \mid \mathcal{C}_{n+1}\right]$ from step 3 to arrive at $\left.\operatorname{Im} \mid M_{2}^{(1)}\right]$.

In evaluating these diagrams we made use of the SCET Feynman rules for soft emission from the Wilson lines $\mathbf{S}_{n_{i}}$, as well as those for the Glauber operators in $O_{G}(x)$, namely 


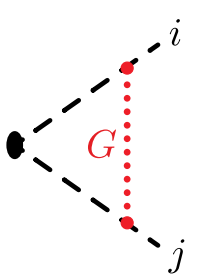

(a)
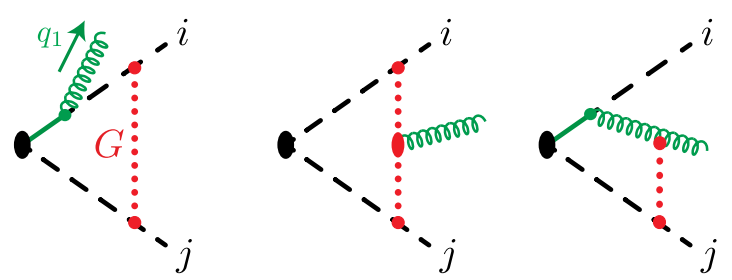

(b)
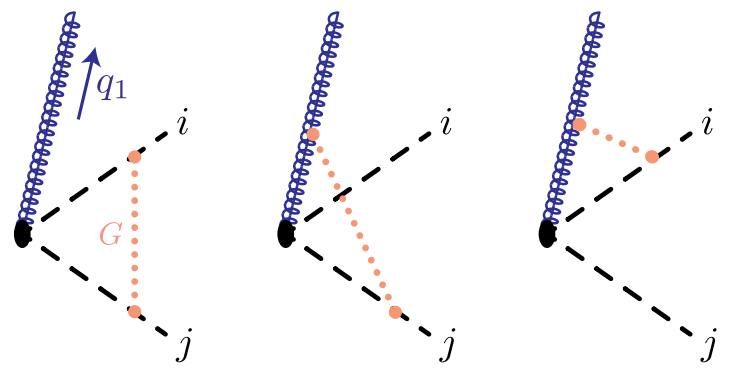

(c)
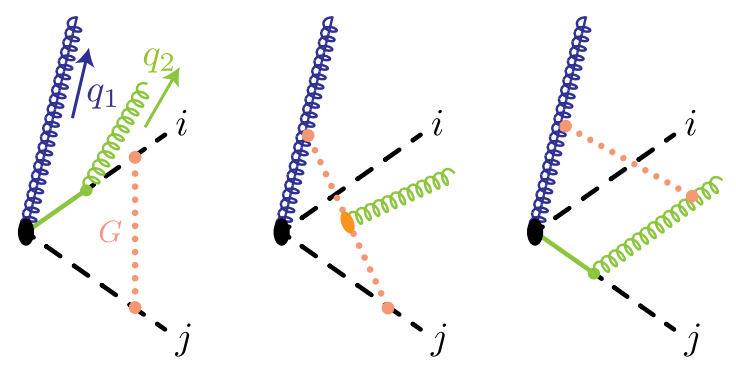

(d)

FIG. 2. Diagrams needed to reproduce the imaginary part of one-loop and up to two real soft gluon emission amplitude. Labels $i, j$ correspond to hard particles. The gluon colored in blue is more energetic of the two $\left(q_{1}\right)$. The green solid lines correspond to off-shell propagators. They have been depicted in this way to make the color ordering apparent. Graphs with Glauber exchange between a soft gluon and its parent are zero in Feynman gauge, and not shown. (a)The Glauber exchange graph in $\mathrm{EFT}_{n}$, (b) Single soft gluon emission graphs with a Glauber exchange in $\mathrm{EFT}_{n}$. The second graph involves the Lipatov vertex. Additional graphs with permutations of $i$ and $j$ are not shown, (c) Glauber exchange graphs in $\mathrm{EFT}_{n+1}$, and (d) An indicative selection of single soft gluon emission graphs with Glauber exchanges in $\mathrm{EFT}_{n+1}$. One must also consider other allowed permutations of gluon 2 and the Glauber vertices.

the collinear-Glauber, the Lipatov vertex and the softGlauber vertices provided in Ref. [8] appearing in Fig. 2(b). The calculation applies to a generic hard scattering operator $O_{n}^{(0)}$. See Ref. [9] for the details.
Step 1 yields

$$
\begin{gathered}
\left.\left.\mid \mathcal{C}_{n}^{(0)}\left(\left\{\omega_{i}\right\}, \mu\right)\right]=\mid M_{0}^{(0)}\right], \\
\left.\left.\operatorname{Im} \mid \mathcal{C}_{n}^{(1)}\left(\left\{\omega_{i}\right\}, \mu\right)\right]=\sum_{i=1}^{n} \sum_{j<i} \overline{\mathbf{C}}^{(i j)}\left(\mu, \sqrt{\omega_{i j}}\right) \mid M_{0}^{(0)}\right],
\end{gathered}
$$

where

$$
\begin{aligned}
\overline{\mathbf{C}}^{(i j)}(m, \mu) & \equiv g^{2} \mathrm{i}\left(\mathbf{T}_{i} \cdot \mathbf{T}_{j}\right) \frac{1}{2} \int \frac{d^{d-2} \ell_{\perp}}{(2 \pi)^{d-2}}\left[\frac{\mu^{2 \epsilon}}{\ell_{\perp}^{2}-m^{2}}\right] \\
& =(-\mathrm{i} \pi) \frac{\alpha_{s}}{2 \pi}\left(\mathbf{T}_{i} \cdot \mathbf{T}_{j}\right)\left(\frac{1}{\epsilon}+\ln \frac{\mu^{2}}{m^{2}}+\mathcal{O}(\epsilon)\right),
\end{aligned}
$$

where $m$ is a gluon mass used to regulate IR divergences, such that the EFT matrix elements are no longer scaleless. The $m$ dependence has canceled in arriving at Eq. (15), such that the Wilson coefficient is IR finite. The EFT matrix elements, being UV divergent, are regulated in dimensional regularization, and hence depend on $\mu$. The $\mathrm{EFT}_{n}$ is matched to the full theory at scales $\mu^{2} \sim\left\{\omega_{i j}\right\}$. In IR and UV finite cases, where the integral in Eq. (16) is bounded so that $\ell_{\perp} \in(a, b)$, we no longer need the gluon mass $m$ and $\overline{\mathbf{C}}^{(i j)}$ becomes

$$
\mathbf{C}^{(i j)}(a, b) \equiv(-\mathrm{i} \pi) \frac{\alpha_{s}}{2 \pi}\left(\mathbf{T}_{i} \cdot \mathbf{T}_{j}\right) \ln \left(\frac{b^{2}}{a^{2}}\right) .
$$

The result of step 2 is that

$$
\begin{aligned}
\left.\operatorname{Im} \mid M_{1}^{(1)}\right] \equiv & \operatorname{Im}\left(\mid\left\langle\left(q_{1}, \varepsilon_{1}\right),\left\{p_{i}\right\}\right| O_{n}\left(\left\{\omega_{i}, n_{i}\right\}\right)\right. \\
& \left.\left.\times \prod_{i=1}^{n} \mathbf{S}_{n_{i}}|0\rangle \mid \mathcal{C}_{n}\left(\left\{\omega_{i}\right\}, \mu\right)\right]\right)^{(1)} \\
= & g \sum_{i=1}^{n}\left(\sum _ { j < i } \left[\mathbf{J}^{(0)}\left(q_{1}\right) \mathbf{C}^{(i j)}\left(q_{1 \perp}^{(i j)}, \sqrt{\omega_{i j}}\right)\right.\right. \\
& \left.+\mathbf{C}^{(i j)}\left(m, q_{1 \perp}^{(i j)}\right) \mathbf{J}^{(0)}\left(q_{1}\right)\right] \\
& \left.\left.\left.+\sum_{j \neq i} \mathbf{C}^{((n+1) j)}\left(m, q_{1 \perp}^{(i j)}\right) \mathbf{d}_{j i}^{(0)}\left(q_{1}\right)\right]\right) \mid M_{0}^{(0)}\right],
\end{aligned}
$$

where $(\ldots)^{(1)}$ indicates evaluating the bracketed expression at one loop. The graphs contributing to Eq. (18) are shown in Fig. 2(b). The Lipatov vertex plays a crucial role in implementing the switch mechanism identified in Ref. [2]: the associated loop momentum is cut off in the IR by $q_{1 \perp}^{(i j)}$ in order to account for production of $q_{1}$, i.e.,

$$
\begin{aligned}
& \int \frac{\mathrm{d}^{d-2} \ell_{\perp}}{\left(\ell_{\perp}^{2}-m^{2}\right)}-\frac{q_{1 \perp}^{2}}{2} \int \frac{\mathrm{d}^{d-2} \ell_{\perp}}{\left(\ell_{\perp}^{2}-m^{2}\right)\left[\left(\ell_{\perp}+q_{1 \perp}\right)^{2}-m^{2}\right]} \\
& \quad=\int \frac{\mathrm{d}^{d-2} \ell_{\perp}}{\left(\ell_{\perp}^{2}-m^{2}\right)} \Theta\left(\left|\vec{\ell}_{\perp}\right|-\left|\vec{q}_{1 \perp}\right|\right),
\end{aligned}
$$

and the non-Abelian structure of the vertex yields commutators of the form $\left[\mathbf{J}^{(0)}\left(q_{1}\right), \mathbf{C}^{(i j)}\left(q_{1 \perp}^{(i j)}, \mu\right)\right]$. The commutators cancel terms from the hard scattering graph which are 
oppositely ordered in transverse momentum. The $\mu$ scale serves as a UV cutoff for the EFT matrix elements, and after combining it with the Wilson coefficients, is replaced by hard scales $\left\{\omega_{i j}\right\}$. The third term results from the rescattering graphs, which are UV finite on their own, and account for the nontrivial frame dependence in the third line in Eq. (7). Equation (18) is in agreement with the calculation presented in Ref. [2] (i.e., Eq. (7) for $N=1$ ). After step 3:

$$
\begin{aligned}
\left.\mid \mathcal{C}_{n+1}^{(0)}\left(\left\{\omega_{1}, \omega_{i}\right\}, \mu\right)\right]= & \left.\mid M_{0}^{(0)}\right] \\
\left.\mathbf{J}^{(0)}\left(q_{1}\right) \operatorname{Im} \mid \mathcal{C}_{n+1}^{(1)}\left(\left\{q_{1 \perp}^{(i j)}, \omega_{i}\right\}, \mu\right)\right]= & \left.\mathbf{J}^{(0)}\left(q_{1}\right) \sum_{i=1}^{n} \sum_{j<i} \mathbf{C}^{(i j)}\left(q_{1 \perp}^{(i j)}, \sqrt{\omega_{i j}}\right) \mid M_{0}^{(0)}\right] \\
& \left.+\sum_{i=1}^{n}\left(\sum_{j<i} \overline{\mathbf{C}}^{(i j)}\left(\mu, q_{1 \perp}^{(i j)}\right) \mathbf{J}^{(0)}\left(q_{1}\right)+\sum_{j \neq i} \overline{\mathbf{C}}^{((n+1) i)}\left(\mu, q_{1 \perp}^{(i j)}\right) \mathbf{d}_{i j}^{(0)}\left(q_{1}\right)\right) \mid M_{0}^{(0)}\right] .
\end{aligned}
$$

The structure of Wilson coefficient in Eq. (20) is analogous to that in Eq. (18) with $m \rightarrow \mu$. This is because the matrix elements in $\mathrm{EFT}_{n+1}$ ought to account for all the IR divergences which cancel in the matching calculation. Each term in Eq. (20) has a physical interpretation: the first term in the second line of Eq. (20) has the same functional form as $\left.\mathbf{J}^{(0)}\left(q_{1}\right) \operatorname{Im} \mid \mathcal{C}_{n}^{(1)}\right]$ [see Eq. (15)], encoding the same UV physics; whereas terms in the third line are new, where it is the quantity $\omega_{1}=q_{1 \perp}^{(i j)}$ that sets the hard scale for $\mathrm{EFT}_{n+1}$.

Finally, step 4 yields

$$
\begin{aligned}
\left.\operatorname{Im} \mid M_{2}^{(1)}\right] \equiv & \left.\operatorname{Im}\left(\left\langle\left(q_{2}, \varepsilon_{2}\right),\left(q_{1}, \varepsilon_{1}\right),\left\{p_{i}\right\}\left|O_{n+1}\left(\left\{q_{1 \perp}^{(i j)}, n_{1}, \omega_{i}, n_{i}\right\}\right)\right| 0\right\rangle \mid \mathcal{C}_{n+1}\left(\left\{q_{1 \perp}^{(i j)}, \omega_{i}\right\}, \mu\right)\right]\right)^{(1)} \\
= & g^{2}\left[\mathbf{J}^{(0)}\left(q_{2}\right) \mathbf{J}^{(0)}\left(q_{1}\right) \sum_{i=1}^{n} \sum_{j \neq i} \mathbf{C}^{(i j)}\left(q_{1 \perp}^{(i j)}, \sqrt{\omega_{i j}}\right)\right. \\
& +\mathbf{J}^{(0)}\left(q_{2}\right) \sum_{i=1}^{n}\left(\sum_{j<i} \mathbf{C}^{(i j)}\left(q_{2 \perp}^{(i j)}, q_{1 \perp}^{(i j)}\right) \mathbf{J}^{(0)}\left(q_{1}\right)+\sum_{j \neq i} \mathbf{C}^{((n+1) i)}\left(q_{2 \perp}^{((n+1) i)}, q_{1 \perp}^{(i j)}\right) \mathbf{d}_{i j}^{(0)}\left(q_{1}\right)\right) \\
& \left.\left.+\sum_{i=1}^{n+1}\left(\sum_{j<i} \mathbf{C}^{(i j)}\left(m, q_{2 \perp}^{(i j)}\right) \mathbf{J}^{(0)}\left(q_{2}\right)+\sum_{j \neq i} \mathbf{C}^{((n+2) i)}\left(m, q_{2 \perp}^{(i j)}\right) \mathbf{d}_{i j}^{(0)}\left(q_{2}\right)\right) \mathbf{J}^{(0)}\left(q_{1}\right)\right] \mid M_{0}^{(0)}\right] .
\end{aligned}
$$

This equation is exactly equal to the imaginary part of Eq. (7) when $N=2$. It is straightforward to extend the result above to the complete result in Eq. (7) by including more tree-level soft gluons.

In conclusion, using SCET we have presented a very compact derivation of the result in Ref. [1] that involved only a handful of diagrams, and where each of the diagrams has a clear physical relevance. The simplicity of this derivation makes the extension to higher orders foreseeable: for example, the two-loop extension will only require the effective one-loop collinear-Glauber, soft-Glauber and one-loop Lipatov vertices. We leave this to future work.

\section{ACKNOWLEDGMENTS}

This work is supported by the UK Science and Technology Facilities Council (STFC) under Grant No. ST/T001038/1.
[1] R. Ángeles Martínez, J. R. Forshaw, and M. H. Seymour, Phys. Rev. Lett. 116, 212003 (2016).
[2] R. Ángeles-Martínez, J. R. Forshaw, and M. H. Seymour, J. High Energy Phys. 12 (2015) 091. 
[3] C. W. Bauer, S. Fleming, and M. E. Luke, Phys. Rev. D 63, 014006 (2000).

[4] C. W. Bauer, S. Fleming, D. Pirjol, and I. W. Stewart, Phys. Rev. D 63, 114020 (2001).

[5] C. W. Bauer, D. Pirjol, and I. W. Stewart, Phys. Rev. D 65, 054022 (2002).

[6] C. W. Bauer and I. W. Stewart, Phys. Lett. B 516, 134 (2001).

[7] C. W. Bauer, S. Fleming, D. Pirjol, I. Z. Rothstein, and I. W. Stewart, Phys. Rev. D 66, 014017 (2002).

[8] I. Z. Rothstein and I. W. Stewart, J. High Energy Phys. 08 (2016) 025.
[9] A. Pathak, arXiv:2108.13440.

[10] A. Bassetto, M. Ciafaloni, and G. Marchesini, Phys. Rep. 100, 201 (1983).

[11] Z. Bern, V. Del Duca, W. B. Kilgore, and C. R. Schmidt, Phys. Rev. D 60, 116001 (1999).

[12] S. Catani and M. Grazzini, Nucl. Phys. B591, 435 (2000).

[13] C. Duhr and T. Gehrmann, Phys. Lett. B 727, 452 (2013).

[14] Y. Li and H. X. Zhu, J. High Energy Phys. 11 (2013) 080.

[15] I. Feige and M. D. Schwartz, Phys. Rev. D 90, 105020 (2014). 